\title{
FILOSOFÍA PARA NIÑOS: UN RETO PARA LA EDUCACIÓN ÉTICA Y EN VALORES
}

\section{PHILOSOPHY FOR CHILDREN: A CHALLENGE FOR ETHICAL AND VALUES EDUCATION}

\author{
Carlos Fernando Velasco Moreno \\ Universidad Santo Tomás - Universidad Abierta y a Distancia \\ Colombia \\ carlosfdovelasco@hotmail.com \\ ORCID: 0000-0001-6132-2052
}

RECIBIDO: 22 DE MARZO DE 2020

ACEPTADO: 1 DE JUNIO DE 2020

Resumen: Esta investigación parte de la necesidad colombiana de desarrollar el pensamiento crítico, principalmente en niños de educación básica primaria, como respuesta a la creciente problemática de conflictos escolares dentro y fuera de las aulas. Esto puede ser atendido desde la implementación de Filosofía para Niños $(\mathrm{FpN})$, porque a partir del análisis de situaciones cotidianas, algunas de ellas convertidas en anécdotas, el docente promueve habilidades de pensamiento que las argumentan y las pasan por el matiz de la crítica. Este proceso se puede articular con las innovaciones que pretende tanto la legislación colombiana, como la educación contemporánea, generando conciencia en el estudiante de soluciones por la vía concertada, llegando a acuerdos y conciliaciones que aborden el conflicto.

Palabras clave: Pensamiento crítico, conflicto escolar, Filosofía para Niños, argumentación, conciencia.

Abstract: This research is based on Colombia's need to develop critical thinking, mainly in primary basic education children, in response to the growing problem of school conflicts in and out of the classroom. This can be addressed from the implementation of Philosophy for Children ( $\mathrm{FpN})$, because from the analysis of everyday situations, some of them turned into anecdotes, the teacher promotes thinking skills that argue them and get them through the nuance of criticism. This process can be articulated with the innovations that both Colombian legislation 
and contemporary education aim, generating awareness in the student of solutions by the concerted way, reaching agreements and conciliations to approach the Conflict.

Key words: Critical thinking, school conflict, Children's Philosophy, argumentation, awareness.

\section{Introducción}

Lo que investigué como estudiante de la Universidad Santo Tomás entre los años 2016 y 2017, fundamentó la implementación del programa de Filosofía para Niños (FpN) en algunas instituciones educativas dentro del nivel de Educación Básica Primaria en los municipios de Chía y Cajicá, en el departamento de Cundinamarca, Colombia. Se sugirió la propuesta FpN como una forma de abordar, desde el diálogo argumentativo, la solución de conflictos escolares que afectan la convivencia y, en algunos casos, el bienestar de los estudiantes en la vida cotidiana, con el ánimo de aprovechar la receptividad de los niños y su potencial desarrollo del pensamiento crítico en el análisis de las situaciones problemáticas.

De lo anterior se desprendieron otros objetivos como la asociación de "solución de conflictos del entorno escolar con la propuesta de FpN para el desarrollo del pensamiento crítico en una ética

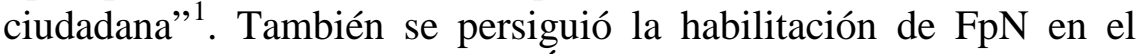
plan de estudios de Educación Ética y en Valores como propuesta didáctica al alcance del docente, y la incorporación de "estrategias metodológicas orientadas a la implementación de la propuesta FpN que comprometan a los docentes en su desarrollo, control y seguimiento" ${ }^{2}$.

\footnotetext{
${ }^{1}$ Primer objetivo específico del Trabajo de investigación titulado en el presente artículo.

${ }^{2}$ Tercer objetivo específico del Trabajo de investigación titulado en el presente artículo.
} 
Con relación al objetivo de la investigación que incentiva una ética ciudadana, Mathew Lipman en su propuesta de FpN resalta el papel de la ética, aporta una definición de la misma que sin duda es una guía para el educador, permitiéndole desenvolverse con mayor solvencia en el análisis ético de las situaciones problemáticas presentadas entre los niños. La define como:

Rama de la filosofía que pretende comprender la conducta moral. Representa una investigación objetiva y desapasionada sobre problemas y situaciones morales. Su fin nunca es adoctrinar, sino ayudar a las personas a que comprendan con mayor claridad cuáles son sus opciones morales y cómo es posible evaluar críticamente esas opciones ${ }^{3}$

El descubrimiento de Harry es la primera novela de Lipman que describe la manera como los niños descubren la filosofía. A partir de allí él construye sus conocidos Manuales Docentes, que son su propuesta pedagógica innovadora en el afianzamiento de FpN, debido a que al final de su obra aclara que son:

Instrumentos de razonamiento básicos, técnicas de pensamiento crítico y de lógica formal e informal que los niños podrán aplicar en cursos superiores a problemas específicos de las diferentes áreas... ofrece un modelo de diálogo entre niños y de éstos con los adultos, tanto en el aula de clase como en la vida familiar o en sus relaciones como amigos ${ }^{4}$

Con la intención de enseñar a pensar sobre lo que se memoriza, FpN realiza su aparición en el análisis que los niños pueden hacer acerca del mundo. Con esto, el maestro se obliga a llenarse de conocimientos que le permitan guiar ese pensamiento, participar en el análisis y gestionar la solución del conflicto, acercándose

${ }^{3}$ LIPMAN, Matthew: Investigación ética - Manual del profesor para acompañar a Lisa, Ediciones de la Torre, Madrid, 1988, pág. 21.

${ }^{4}$ LIPMAN, Matthew: El descubrimiento de Harry, Ediciones de la Torre, Madrid, 1988, pág. 72. 
positivamente a la formación ético-ciudadana. Adela Cortina, en su artículo Resolver los conflictos, hacer la justicia, hace una meditación sobre la forma como el docente aborda la resolución de conflictos desde la ética ciudadana:

Educar en la resolución de conflictos, significa enseñar a la persona a enfrentarlos con la voluntad positiva de no permitir que le arrebaten la capacidad de ser dueña de sí misma. Y es materia indispensable en la educación porque, sin la habilidad suficiente para hacerlo, la persona sufrirá a lo largo de su vida situaciones de dolor inevitables. ${ }^{5}$

Además, ella considera que el rol formativo del maestro no se detiene en la educación para la resolución de conflictos, por el contrario, debe enriquecer el panorama del estudiante desde el conocimiento ético. La ética ciudadana, en su accionar filosófico, le permite a los niños: "encontrar soluciones justas para los que se encuentren implicados. Porque las relaciones humanas se producen entre sujetos que, aun "antes" de saberse en confrontación, se reconocen mutuamente como seres humanos" ${ }^{\prime 6}$

\section{Aproximación al contexto educativo}

A pesar de los esfuerzos en la enseñanza de la cátedra de Ética, fundamentada en cuestiones como las normas familiares y la escolaridad, a los estudiantes se les dificulta actuar en la vida cotidiana dentro de un ambiente de convivencia sano. Teniendo como punto de partida el hecho que el acto propio afecta directa o indirectamente a las personas que rodean a quien lo ejecuta, aparecen situaciones que en algunas ocasiones generan conflictos.

${ }^{5}$ CORTINA, Adela: "Resolver los conflictos, hacer la justicia”, en Cuadernos de pedagogía, número 257, Madrid, 1999, pág. 2.

${ }^{6}$ CORTINA, Adela: Educación en valores y Responsabilidad cívica, Editorial el Búho Ltda, Bogotá, 2005, pág. 151. 
La observación de comportamientos especialmente en los niños de grado $5^{\circ}$ de primaria genera interés en directivos y maestros, por ser una población que asume el rol de ser la mayor en su nivel y por ser un punto de transición hacia la educación básica y media. Se encuentran en una etapa escolar que solicita un pensamiento crítico y que permite tomar decisiones a partir de una reflexión filosófica adquirida a través de la formación ético-social. Una formación que será incorporada por ellos y que determinará su comportamiento. Por lo tanto, consideramos que es necesario proponer la incorporación de FpN dentro del área de Educación Ética y en Valores, ya que esta disciplina:

Busca fomentar y conservar en los niños y adolescentes la actitud que tienen en común los filósofos con la infancia: la curiosidad por todo lo que les rodea y el no dar nada por sentado. La inacabable cadena de preguntas " $\dot{\mathrm{C}} Y$ por qué? ¿Y por qué? ¿Y por qué?” que caracteriza una determinada etapa del desarrollo del niño se convierte en modo de vida para el filósofo, y esa es la actitud que se busca salvaguardar ${ }^{7}$

Los niños de este grado han recibido, en los años anteriores, formación en Educación Ética y Valores; de manera que han desarrollado la habilidad de elaborar juicios valorativos frente a situaciones conflictivas que hacen parte de la vida cotidiana. Estos muchas veces pueden calificarse de drásticos, es decir, que asumen la sanción en otros especialmente cuando no les afecta personalmente. No suelen aceptar un término medio en las apreciaciones respecto a los demás, lo que debe ser aprovechado para fundamentar y enriquecer una construcción de juicios asertivos, que incluyan mayor variedad de posibilidades para analizar las situaciones conflictivas, evitando los juicios excesivamente radicales.

7 MORENO ARTIDIELLO, Mabel Marta: "Filosofía para Niños y Niñas (FPNN): una oportunidad diferente para pensar en la escuela”, en Ciencia y Sociedad, número 43, Santo Domingo, 2018. Pág 27. 
Al observar el plan de estudios en primaria, hay un contenido prefilosófico que inicialmente aporta conceptualización y acercamiento a la realidad del estudiante, esto se evidencia en la siguiente tabla ${ }^{8}$ :

\begin{tabular}{|l|l|l|l|}
\hline PRIMERO & SEGUNDO & TERCERO & CUARTO \\
\hline -Actos & - Conceptualización & - ¿Quién soy? & -Conciencia \\
buenos y & básica de la ciencia & - Normas en mi & -Conciencia de sí \\
actos malos. & ética. & familia y en mi & mismo. \\
- Me respeto & - Esquema & Colegio. & - Deber y norma \\
a mí mismo & corporal. & - Conflicto. & - Leyes \\
y a los & - ¿Cómo reconocer & - Mis deberes & naturales. \\
demás. & actos buenos y & básicos para la & - Leyes positivas. \\
-Cualidades & actos malos? & convivencia & -Voluntad, \\
en los & - Acercamiento al & social. & temperamento y \\
demás. & concepto de & - Acercamiento al & carácter. \\
- Gratitud & conciencia moral & concepto de & -Reconocimiento \\
- Solidaridad & - Valores éticos y & intencionalidad & de los demás \\
- Paz. & morales. & moral. & -Cuidado del \\
- Cuidado & - Respeto & - Diversidad y & medio ambiente. \\
con los seres & - Responsabilidad. & pluralidad: & \\
de la & - Honestidad & respeto, tolerancia, & \\
naturaleza. & - Formación de la & solidaridad. & \\
& voluntad & & \\
\hline
\end{tabular}

Hay temas filosóficos que pueden ser aprovechados por el docente para desarrollar FpN. Estos se presentan a medida que se va ascendiendo de grado. Los contenidos de las asignaturas pertenecientes al área de Humanidades se van complejizando e intensificando, como puede observarse en la siguiente gráfica ${ }^{9}$.

8 Adaptación propia con información tomada de los Planes de Estudio de Educación Ética y en Valores del colegio Celestin Freinet de Chía, Cundinamarca (2017).

${ }_{9}^{9}$ Adaptación propia con información tomada de los Planes de Estudio del área de Humanidades del colegio Celestin Freinet de Chía, Cundinamarca (2017). 


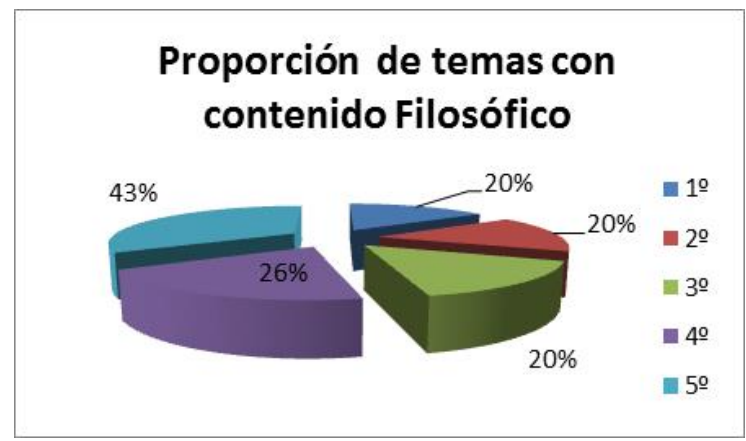

Por otro lado, al revisar los registros disciplinarios de los estudiantes, se encuentran algunos conflictos escolares considerados típicos por docentes, coordinadores y padres de familia. Entre ellos se observan los relacionados con la transgresión del Manual de Convivencia institucional, coincidiendo con los más reportados por los docentes:

\begin{tabular}{|l|l|}
\hline Casos más frecuentes $^{\mathbf{1 0}}$ & Porcentaje \\
\hline - Incumplimiento de tareas. & $25 \%$ \\
\hline - Impuntualidad & $15 \%$ \\
\hline - Bullying & $15 \%$ \\
\hline - Agresividad & $15 \%$ \\
\hline - Irrespeto a la autoridad & $10 \%$ \\
\hline - Fraude & $6 \%$ \\
\hline -Problemas con redes sociales. & $5 \%$ \\
\hline - Daños en pupitres & $5 \%$ \\
\hline $\begin{array}{l}\text { - Faltas leves (pequeños brotes de indisciplina, porte de } \\
\text { uniforme) }\end{array}$ & $4 \%$ \\
\hline
\end{tabular}

Lo último que hice para el análisis del contexto educativo, fue una encuesta a 37 estudiantes de Grado Quinto de Educación Básica Primaria. El objeto de la misma fue hacer un diagnóstico de las disposiciones asumidas por los niños frente a los conflictos

${ }^{10}$ Elaboración propia con información tomada de los observadores estudiantiles. 
cotidianos; así mismo, mediante este ejercicio se evalúa la pertinencia del programa de $\mathrm{FpN}$ como estrategia didáctica para abordar la solución de conflictos desde el diálogo en comunidad, la reflexión y el análisis.

\begin{tabular}{|l|l|l|l|l|}
\hline No. & PREGUNTAS & S & N & NS/NR \\
\hline 1. & $\begin{array}{l}\text { ¿Puedes identificar en tu familia, en el } \\
\text { colegio o en tu grupo de amigos cuando } \\
\text { hay un problema o conflicto? }\end{array}$ & 31 & 4 & 2 \\
\hline 2. & $\begin{array}{l}\text { Ante los problemas o conflictos ¿tratas } \\
\text { siempre de solucionarlos personalmente? }\end{array}$ & 25 & 8 & 4 \\
\hline 3. & $\begin{array}{l}\text { ¿Haces reclamos a las personas sobre los } \\
\text { conflictos que ellos te generan? }\end{array}$ & 23 & 9 & 5 \\
\hline 4. & $\begin{array}{l}\text { ¿Algunas veces has resuelto conflictos con } \\
\text { peleas o agresividad? }\end{array}$ & 10 & 22 & 5 \\
\hline 5. & $\begin{array}{l}\text { ¿Algunas veces a los conflictos has } \\
\text { reaccionado con groserías? }\end{array}$ & 5 & 30 & 2 \\
\hline 6. & $\begin{array}{l}\text { ¿Has hecho cosas “contra los demás”, } \\
\text { (hablar mal, zancadilla, balonazos, etc.) } \\
\text { cuando tienes un conflicto con ellos? }\end{array}$ & 8 & 26 & 3 \\
\hline 7. & $\begin{array}{l}\text { Antes de dar solución a un conflicto ¿has } \\
\text { reflexionado sobre la mejor forma de } \\
\text { hacerlo? }\end{array}$ & 25 & 8 & 4 \\
\hline 8. & $\begin{array}{l}\text { ¿Con tus compañeros te has puesto de } \\
\text { acuerdo para solucionar conflictos? }\end{array}$ & 32 & 2 & 3 \\
\hline 9. & $\begin{array}{l}\text { Después de tener un conflicto ¿̇has } \\
\text { analizado el por qué se dio? }\end{array}$ & 28 & 4 & 5 \\
\hline 10. & $\begin{array}{l}\text { ¿Les cuentas a tus papás o profesores los } \\
\text { conflictos que tienes con los demás? }\end{array}$ & 32 & 2 & 3 \\
\hline
\end{tabular}

${ }^{11}$ Cuestionario del manejo del conflicto antes de la aplicación de FpN. Allí se especifica en cada pregunta tres opciones de respuesta: S (si) N (no) NS/NR (no sabe, no responde). 


\section{Gráfica de barras $^{12}$}

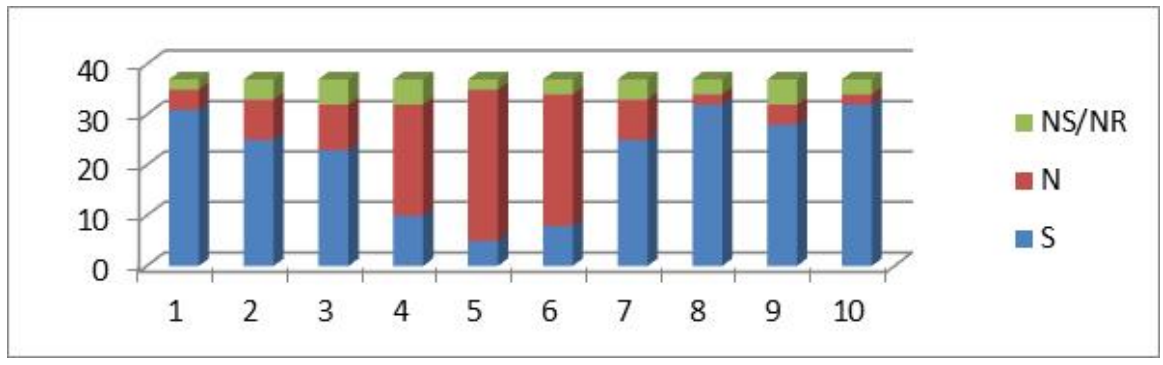

Como se observa en el gráfico anterior, los niños identifican conflictos que asocian con actitudes de mala cara, preocupación, discusión, gritos, grosería, aislamiento. Con la respuesta en el interrogante número 2, se muestra la autonomía que el estudiante quiere tener al solucionar personalmente sus problemas. Sin embargo, hay una dificultad en la aceptación de algunas soluciones impulsivas: agresividad, grosería o gestos que los estudiantes intentan explicar de involuntarios cuando realmente no lo son. Se evidencia prevención en las preguntas 3, 4, 5, 6 y 7, un querer dar la respuesta acorde a lo que el docente desea escuchar.

Por otra parte, la respuesta a la pregunta 8 es un indicador que requiere acciones preventivas ofrecidas por $\mathrm{FpN}$, especialmente la comunidad de indagación, ya que en ella se ve la tendencia a buscar soluciones entre sus miembros. En general, la encuesta manifiesta la necesidad de un espacio de diálogo, análisis y reflexión en los conflictos escolares que aparecen en la cotidianidad.

${ }^{12}$ Gráfica de la encuesta, en donde el color azul representa las habilidades que los estudiantes deberían tener para el abordaje de los conflictos. 


\section{Manejo del conflicto con FpN}

Posteriormente incorporé la propuesta de FpN. Se aplicó el ejercicio de identificar y evaluar algunas situaciones problemáticas para conocer las interpretaciones de los estudiantes, utilizando algunas anécdotas de hechos sucedidos en la institución y otras conocidas como docente, en mi trayectoria pedagógica. Seguido a esto, establecí pequeñas comunidades de indagación para que los mismos estudiantes propusieran soluciones o maneras en las que ellos habrían actuado en circunstancias parecidas; se socializaron las respuestas para analizar las soluciones óptimas que no vulneraran a los demás, lo que contribuyó a la generación de un vocabulario básico que permitió abordar mejor la FpN dentro de dichas comunidades.

En mi experiencia docente, la aplicación de FpN por medio de talleres, me ha hecho sentir comprometido desde el inicio del proceso de preparación de los mismos. Debo pensar y proyectar el ambiente en el que se darán, por ello algunas veces influyen elementos que hacen parte de la decoración del aula de clase, para recrear escenarios externos dados al desarrollo de las anécdotas o narraciones que se utilizarán en las sesiones de trabajo. En la competencia crítica, me interesó realizar un proceso de aprehensión, es decir, una apropiación consciente del conocimiento que se pretende adquirir. Utilizo como punto de partida una narración, algunas veces de la cotidianidad y otras de textos existentes, que permita hacer una serie de preguntas que no solamente constituyen una reconstrucción literal, sino que estimulan el desarrollo del pensamiento crítico cuando son extensivas a actitudes, apropiación de conceptos y afirmación de posturas frente a las situaciones presentadas.

Al analizar los manuales escritos por Diego Pineda para una posterior implementación, novelas como clínica de muñecas, Lisa y Harry tienen en cada pasaje un comentario que aclara los conceptos 
que están implícitos; también hay unos planes de diálogo que son una serie de preguntas orientadoras que ayudan a explorar esos conceptos para identificarlos y distinguirlos, y así poder aplicarlos en ciertos contextos. Finalmente, en dichos manuales hay una serie de actividades de recreación conceptual, allí aparecen actividades lúdicas, corporales y de escritura.

Luego de varias sesiones transcurridas entre septiembre de 2016 y junio de 2017, se aplicó al mismo grupo de 37 estudiantes, del grado quinto de básica primaria, una encuesta que permite observar los avances en las disposiciones y habilidades del pensamiento crítico de FpN.

\begin{tabular}{|l|l|l|l|l|}
\hline No. & PREGUNTAS & S & N & NS/NR \\
\hline 1 & $\begin{array}{l}\text { ¿Consideras que una forma de resolver los } \\
\text { conflictos es analizar antes de tomar } \\
\text { acciones? }\end{array}$ & 37 & 0 & 0 \\
\hline 2 & $\begin{array}{l}\text { ¿Crees que puedes construir historias o } \\
\text { anécdotas a partir de los conflictos que se } \\
\text { presentan en tu colegio? }\end{array}$ & 24 & 8 & 5 \\
\hline 3 & $\begin{array}{l}\text { ¿Consideras importante el sentarte a dialogar } \\
\text { con tus profesores y compañeros los } \\
\text { conflictos que hay en tu colegio? }\end{array}$ & 30 & 5 & 2 \\
\hline 4 & $\begin{array}{l}\text { ¿Consideras que las soluciones que se van } \\
\text { dando a cada conflicto se convierten en } \\
\text { experiencias valiosas para solucionar otros? }\end{array}$ & 31 & 4 & 2 \\
\hline 5 & $\begin{array}{l}\text { ¿Podrías hacer un registro de anécdotas } \\
\text { valiosas y de soluciones a los conflictos que } \\
\text { se presenten en tu curso? }\end{array}$ & 22 & 8 & 7 \\
\hline
\end{tabular}

\footnotetext{
${ }^{13}$ Cuestionario del manejo del conflicto después de la aplicación de FpN. Allí se especifica en cada pregunta tres opciones de respuesta: S (si) N (no) NS/NR (no sabe, no responde).
} 


\section{Grafica de barras $^{14}$}

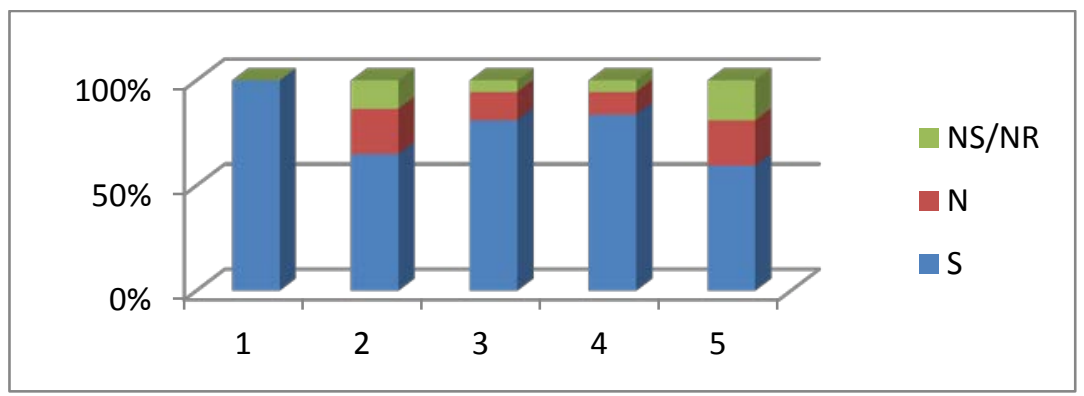

$\mathrm{Al}$ revisar el impacto inicial, se evidencia en los niños gusto por la búsqueda de la solución de conflictos por medio del diálogo y el análisis, ello por el ejercicio que se realiza de buscar alternativas y generar las más adecuadas. Llama la atención de los niños la construcción de anécdotas y el reto de registrarlas en un cuaderno o por medio de grabaciones, pues a partir de ellas surgen mecanismos de solución.

En esta encuesta, también incorporaré preguntas abiertas con el fin de identificar de una forma directa su pensamiento crítico en relación con la propuesta FpN. A continuación, se observan algunas respuestas ${ }^{15}$ significativas:

\footnotetext{
${ }^{14}$ Gráfica que arroja los resultados del abordaje de los conflictos por parte de los estudiantes, luego de haber recibido la aplicación de los talleres de FpN. El color azul simboliza el impacto positivo que FpN obtuvo en el grupo.

${ }^{15}$ Pensamiento de los estudiantes frente a la relación de FpN con la resolución de conflictos escolares dentro de una ética ciudadana.
} 


\begin{tabular}{|c|c|c|}
\hline ¿Qué es para ti FpN? & $\begin{array}{l}\text { ¿Cómo la FpN colaboraría en } \\
\text { la convivencia escolar? }\end{array}$ & $\begin{array}{l}\text { ¿Qué es lo que más te } \\
\text { gusta de la clase de } \\
\text { ética? }\end{array}$ \\
\hline $\begin{array}{l}\text { - Una filosofía que nos } \\
\text { pone a pensar } \\
\text { mediante un dialogo. } \\
\text { - Nos ayuda a pensar } \\
\text { cómo resolver los } \\
\text { conflictos en el } \\
\text { colegio, en la casa y } \\
\text { los grupos. } \\
\text { - Filosofía para niños, } \\
\text { para mí es una forma } \\
\text { de entrar en razón. } \\
\text { - Una forma de hacer } \\
\text { filosofía a los niños } \\
\text { con historias que les } \\
\text { ayudan en el futuro. } \\
\text { - Filosofía para niños: } \\
\text { es una forma de } \\
\text { aprender y evitar los } \\
\text { conflictos en el colegio } \\
\text { y en la vida diaria. }\end{array}$ & $\begin{array}{l}\text { - Porque en un conflicto se } \\
\text { piensa si se puede solucionar. } \\
\text { - Pensando antes de actuar. } \\
\text { Para que cuando alguien te } \\
\text { hace algo no te gane la } \\
\text { venganza. } \\
\text { - Para saber cómo reaccionar } \\
\text { ante problemas de la vida y en } \\
\text { un futuro tal vez muy cercano. } \\
\text { - Aprender a dialogar y a } \\
\text { analizar los problemas. } \\
\text { - Me gustaría que colocaran } \\
\text { FpN en el colegio. Nosotros } \\
\text { como estudiantes } \\
\text { aprenderíamos a manejar los } \\
\text { conflictos en nuestro colegio. } \\
\text { - Nos ayuda a resolver los } \\
\text { conflictos y peleas. } \\
\text { - Ayudarnos a no pegarnos } \\
\text { cuando peleamos. }\end{array}$ & $\begin{array}{l}\text { - Aprender a convivir } \\
\text { con los demás. } \\
\text { - Los valores como: } \\
\text { respeto, paz, fortaleza } \\
\text { y tolerancia. } \\
\text { - Nos ayuda a saber } \\
\text { cómo debemos actuar. } \\
\text { - Los derechos y los } \\
\text { deberes. } \\
\text { - Aprender a vivir en } \\
\text { comunidad. } \\
\text { - Que nos enseñan a } \\
\text { ser mejores personas } \\
\text { en muchas } \\
\text { circunstancias o o } \\
\text { problemas de la vida } \\
\text { de cada quien. } \\
\text { - Las historias y saber } \\
\text { qué es FpN. }\end{array}$ \\
\hline
\end{tabular}

\section{Hallazgos con algunas entrevistas}

Como investigador solamente realicé dos entrevistas a personas directamente relacionadas con la formación de los estudiantes en el terreno de la ética ciudadana dentro de la misma institución. La primera persona fue la psicóloga escolar Diana Amórtegui, a quien le pregunté cuál era su opinión en relación con la incorporación de contenidos a planes de estudio relacionados con la vida cotidiana del estudiante. Desde su experiencia como orientadora y testigo de algunos procesos curriculares, considera que la cercanía de las materias de corte humanista con acontecimientos cotidianos, desarrolla sensibilidad en el estudiante y lo hace protagonista de su 
propia historia; así mismo le permite ser más crítico y argumentativo con las causas y consecuencias de sus propias acciones.

El proceso de crecer y aprender de la propia experiencia y la de los demás, es enriquecedor para la historia personal de vida de los niños y niñas; sin embargo,

Este proceso lleva tiempo y también lleva tiempo formar una comunidad de búsqueda. No puede esperarse en $\mathrm{FpN}$ que la comunidad de búsqueda se establezca en una sesión, en una semana, o en un mes. La experiencia intelectual y social se da en el tiempo, y el tiempo que se dedica a trabajar con los niños en un proceso de indagación filosófica. En un momento dado, una comunidad de búsqueda llega a ser una forma de vida ${ }^{16}$.

La psicóloga considera que la incorporación de conocimientos que se conviertan en herramientas estratégicas de solución, contribuirían de manera significativa al interior del aula o de la vida escolar. Seguramente traería como consecuencia, mayor satisfacción y atención oportuna de los casos en los cuales priman los acaloramientos u opiniones subjetivas de alguna de las partes que participan o influyen en los conflictos. Así, el estudiante pasa a ser autogenerador de soluciones efectivas.

Respecto a lo anterior, Mónica Velasco quiere resaltar la labor concreta y cuidadosa del docente por incentivar el crecimiento de los estudiantes, pues dentro de los objetivos del programa FpN se ubica el

Desarrollar personas autónomas, capaces de pensar por ellas mismas acerca de cosas que les son importantes. Pero hay que entender correctamente autonomía. Lipman señala que la noción de autonomía debe ser considerada con precaución porque existe el

\footnotetext{
${ }^{16}$ VELASCO, Mónica: "Filosofía para niños: una alternativa para resignificar la práctica”, en Sinéctica, número 8, Guadalajara, 1996, pág. 12.
} 
peligro de que un individuo o grupo autónomo piense sólo para sí mismo ignorando la influencia de otros contextos ${ }^{17}$

Cuando le pregunté por las estrategias pedagógicas que deberían estar presentes en la propuesta temática de Ética y Valores humanos para reforzar la solución de conflictos, según su criterio y experiencia de orientación personal, me dijo que resultarían indispensables todas aquellas que contribuyan a reforzar valores como el respeto, la tolerancia, la convivencia y la amistad entre los estudiantes. Resultan muy oportunas aquellas estrategias pedagógicas que orientan el comportamiento y que incentivan a los niños y niñas a desarrollar habilidades de pensamiento que les permitan desenvolverse en análisis de casos, mesas redondas, entrevistas, reconstrucción de anécdotas, elaboración de carteleras, todo esto encaminado a la búsqueda de alternativas, no a desaparecer el conflicto, porque éste hace parte de la dinámica escolar permanente.

El desarrollo de las actividades propuestas por la orientadora escolar para capacitar al estudiante, cobra mayor sentido al articularse con el objetivo de esta investigación, impulsar las habilidades del pensamiento crítico. Mónica Velasco no solo las específica, sino que deja claro que hacen que FpN se concentre en Desarrollar un buen juicio, lo que significa, desarrollar la habilidad de pensar bien, de dar razones fundamentadas en criterios y desarrollar sensibilidad al contexto. Para alcanzar este objetivo, la clase tiene que transformarse en una comunidad de búsqueda donde se desarrollen habilidades de razonamiento y de indagación como:

- Presentar razones para sostener los puntos de vista.

- Preguntar a otros para que expliquen lo que quieren decir.

- Elaborar inferencias a partir de lo que se expone.

- Ser sensible al contexto en el cual se elaboran juicios.

- Emplear criterios consistentes y relevantes.

${ }^{17}$ Ibíd., pág. 8. 
- Darse cuenta del propio proceso de pensamiento y poder autocorregirse.

- Demostrar disposición para modificar los propios puntos de vista.

- Formular preguntas relevantes.

- Utilizar razonamiento hipotético.

- Identificar las implicaciones de aceptar o no un punto de vista.

- Poder generalizar en forma justificada. ${ }^{18}$

Las anteriores habilidades de pensamiento están presentes inexorablemente en el proceso de toma de decisiones de los niños. Como herramientas cognitivas les permiten hacer un análisis de las situaciones problemáticas, conduciéndolos a tomar cierta distancia de ellas para generar soluciones apropiadas y eficaces. Desde luego, esto se lleva a cabo haciendo uso del dominio de la escucha y del habla, debido a que entre más las dominen, más rápido se adaptarán a la filosofía mediante razonamientos y juicios coherentes. Por ende,

Cualquier niño que es capaz de usar un lenguaje inteligible es capaz de ser educado y de crecer, y por tanto es capaz de la clase de discurso y conversación que la filosofía implica. La meta no es hacer de los niños pequeños filósofos, sino ayudarlos a pensar mejor de como lo hacen ahora. $^{19}$

Los pedagogos Dewey y Vygotsky recomiendan FpN, enfatizan en la necesidad de enseñar a pensar y no solo a memorizar. Que los niños recuerden lo que se les ha dicho, no es suficiente, deben examinar y analizar ese material. Pensar es el procesamiento en el que los niños aprenden acerca del mundo con los sentidos, eso también hay que aplicarlo en la escuela. Por ende, en vez de memorizar, a los niños se les deben brindar bases de razonamiento, juicio y formación de conceptos. Ello hace más dinámico el trabajo

\footnotetext{
${ }^{18}$ Ibíd., pág. 5.

${ }^{19}$ NAJÍ, Saeed: “Filosofía para niños entrevista a Matthew Lipman”, en Tedium Vitae, número 2, Guadalajara, 2012, pág. 4.
} 
de la clase y obliga al docente a estar en permanente de actualización, siempre con el ánimo de contribuir positivamente a la madurez del pensamiento.

Sobre la solución de conflictos fundamentada en conocimientos del área de Ética y Valores, la psicóloga piensa que formar en ética ciudadana es el reto del educador contemporáneo. Ve con interés particular la inclusión que se hizo de esta temática y el impacto que su implementación trajo en los procesos de apropiación de conocimientos en los estudiantes, pues a partir allí, se podrá hacer un ejercicio importante de fundamentación para los docentes del área, quienes habrán de comprender que en la dinámica comunitaria formativa del ciudadano en FpN, la inmadurez se vive como un estado de crecimiento positivo del niño.

Además de las habilidades de razonamiento y de las actitudes y habilidades de discusión hay un componente afectivo de gran importancia: el mutuo cuidado de los diferentes miembros del grupo, la disposición de verse transformado por el otro, y el desarrollo de una confianza básica respecto a los otros miembros que ayuda a que cada miembro tome su lugar en el mundo y lo valore. Este componente es una condición para desarrollar la autoestima, y es precisamente lo que hace posible que el niño experimente su inmadurez y su dependencia como una fuerza positiva. ${ }^{20}$

Como último interrogante de la entrevista con la psicóloga le pedí que enunciara los 3 conflictos más recurrentes en el área de orientación. El primero sería la excesiva dependencia de muchos niños por acudir permanentemente al padre de familia para que sea éste quien resuelva situaciones leves, que podrían solucionarse desde un ejercicio reflexivo. El segundo, entra de alguna manera en contraposición con el anterior, es el temor a la sanción o a ser juzgados por las familias; consiste básicamente en ocultar situaciones problema, lo cual no resuelve, sino que acrecienta el conflicto. Finalmente, el tercero alude al manejo de la mentira,

${ }^{20}$ VELASCO, Mónica op. cit., pág. 8. 
fraude y evasión de responsabilidades, que requiere de una profunda reflexión ética para llevar al niño hacia el abandono de este proceder.

El acercamiento con los que tienen mayor relación con los niños, me permitió entender mejor la validez de FpN dentro de la investigación. Sustenta la propuesta de generar conciencia en el estudiante para llegar a conciliaciones que aborden el conflicto, cumpliendo con las exigencias de la educación contemporánea, entre las cuales está la participación en una construcción curricular que debe reconocer inicialmente la posición de los niños frente al conocimiento filosófico, la cual se basa en el supuesto de que ellos mismos "tienen una disposición natural para pensar acerca de su vida cotidiana y de los problemas que ésta les plantea y que además están deseosos de darle sentido a su experiencia”21

Lo anterior conduce a que desde la práctica docente debería aprovecharse el potencial que tiene hacer FpN para, por una parte, darle prioridad a la aprehensión de habilidades de pensamiento crítico como la identificación y el análisis; y por la otra, se reflexione junto a los niños sobre situaciones cotidianas que les resultan problemáticas, para que sean ellos quienes identifiquen causas, origen del conflicto y posibles consecuencias. Este aprovechamiento pedagógico hace que se remitan al conocimiento filosófico por medios didácticos, encontrando allí las soluciones adecuadas, insistiendo en la postura ética ciudadana constructiva. El departamento de Orientación Escolar en las instituciones educativas es fuente de consulta y de seguimiento al desempeño de la personalidad de los educandos, por lo que aporta preciada información acerca de los casos en los que se identifican conflictos escolares. Amórtegui los aborda desde la perspectiva psicopedagógica. Este esfuerzo, atendiendo de manera inmediata y oportuna los casos, refleja alternativas de solución por diferentes

${ }^{21}$ Ibíd., pág. 5. 
medios: entrevistas con estudiantes, con padres de familia, remisiones a terapeutas, pruebas y seguimiento. Sin embargo, con FpN su labor no estaría envuelta en un exceso de trabajo, sino que sobre ella recaerían, en mayor medida, los conflictos escolares graves que no se pudieron abordar desde las habilidades de pensamiento de los agentes directamente involucrados.

Lo anterior cobra mayor validez cuando tiene una finalidad moral, que le permite al niño reconocerse a sí mismo y a los demás como sujetos morales que se encuentran en permanente interrelación. Valorar y respetar individualidades, desde una óptica moral, genera una reflexión permanente alrededor de los actos humanos, llevando al niño a estudiar alternativas de solución de diferentes problemas ético-ciudadanos. Pues en la medida en que el niño reconozca y analice cada una de ellas, estará madurando su pensamiento filosófico. No se pueden contemplar las decisiones tomadas a la ligera, sino que es necesario ir más allá; es decir, identificar en un conflicto escolar todas las variables posibles, empleando criterios conscientes y relevantes para optar por aquella que se ajuste a una realidad moral colectiva, en la que todos los implicados manifiesten un estado de satisfacción.

Pasando a la segunda entrevista, el docente de ética Javier Gómez del colegio Celestin Freinet, aportó en gran medida para clarificar los objetivos de mi investigación. Respecto al proceso que él mismo sigue para revisar un plan de estudios, conservando contenidos, formulándolos, y de ser necesario, revaluándolos, afirma que generalmente se acude a la propuesta preestablecida que tiene la institución. Sin embargo, por las exigencias curriculares contemporáneas en cuanto a las innovaciones que se han hecho a los Planes Estratégicos de Educación Nacional, año tras año, es necesario ver la trazabilidad del área de Educación Ética y en Valores Humanos con estas novedades, para luego compararlas con los temas desarrollados hasta el momento. Esto con el ánimo de descubrir la correlación que haya con las otras áreas o asignaturas y 
de evitar los contenidos repetitivos que no se ajusten a las necesidades del presente educativo.

La ética, ante todo, es una disciplina filosófica. Al hacer parte la filosofía de las dimensiones del ser humano, no puede ser, de ninguna manera, relegada a una clase de una hora semanal, sino que debe iluminar la convivencia escolar e igualmente las áreas del conocimiento. Estanislao Zuleta, un filósofo y pedagogo colombiano, hace una crítica a la educación nacional:

Además del problema de enseñar resultados, sin enseñar los procesos del conocimiento, existe un problema esencial: en la escuela se enseña sin filosofía y ese es el mayor desastre de la educación. Se enseña geografía sin filosofía, biología sin filosofía, historia sin filosofía y filosofía sin filosofía $^{22}$

Al desarrollar el plan de estudios de Educación Ética y en Valores, el docente procura tener en cuenta la visión filosófica que compromete el área misma. Inicialmente hubo dificultad, ya que ésta, de alguna manera, carecía de identidad y se confundía con Religión porque, según la ley colombiana del siglo pasado, se llamaba Educación Ética y Religiosa. Los temas se cruzaban y muchas de las normas que se enseñaban eran llevadas de la mano junto a las prácticas religiosas. Así mismo, ocurrían confusiones con Urbanidad, Cívica y otras disciplinas. El MEN (Ministerio de Educación Nacional) ha publicado innovaciones curriculares que incorporan temas filosóficos, obligando a los niños a tomar posición frente al desarrollo temático. Esta situación exige un pensamiento crítico estructurado, que es precisamente el aporte de FpN dentro del área.

Cuando le pregunté sobre la utilidad de la implementación de contenidos filosóficos en el área de Educación Ética y en Valores,

22 ZULETA, Estanislao: Educación y democracia, un campo de combate, editorial Corporación Tercer Milenio, Bogotá, 1985. Pág. 25. 
Javier consideró que ésta se da desde los mismos conocimientos que incentivan en el educando la capacidad analítica, además de su objetividad frente a diversas problemáticas sociales. Estos contenidos lo inducen a ser crítico, asumiendo y afrontando la cuestión central del conflicto. Esto es así, porque uno de los supuestos básicos de la investigación es que los niños son, en potencia, interlocutores racionales competentes, por lo que pueden y deben intervenir en discusiones racionales. Desde ese punto de vista, la filosofía también puede aportarles mucho en su formación. En todos los niveles de escolaridad, con la inclusión de la filosofía en la totalidad del currículo educativo:

El cultivo o la enseñanza apropiados de la filosofía, por la naturaleza misma de la disciplina, implican ya una manera de razonar y de actuar que, podríamos decir, promueve tanto el crecimiento personal e interpersonal como la formación de valores democráticos para la convivencia ciudadana. $^{23}$

El docente considera que los contenidos filosóficos introducidos en Ética y Valores, deben pensarse sobre las necesidades detectadas en los grupos desde el año inmediatamente anterior. Esto sin dejar de lado las que permitan identificar las conductas iniciales de los estudiantes, atendiendo al dinamismo que tiene el proceso pedagógico de FpN y a la flexibilidad curricular. De igual manera, son pertinentes en su implementación aquellas necesidades que, en determinado momento, se ajustan a las características especiales de cada curso.

Javier agrega que las estrategias metodológicas de FpN son claves en el proceso enseñanza-aprendizaje, porque ellas se convierten en habilidades de pensamiento crítico óptimas para la vida escolar. Él

${ }^{23}$ VALDÉS HOYOS, Diana: "Filosofía para niños y lo que significa una educación filosófica”, en Discusiones Filosóficas, número 16, Manizales, 2011. Pág. 162. 
propone reconstrucción de historias, elaboración de grupos de trabajo y conversatorios que aborden un problema, lo analicen y evidencien alternativas de solución pensadas por los mismos niños. Después, le pregunté por los conflictos que pueden ser tratados desde una fundamentación filosófica y que requieren de mayor atención. Él enunció algunos como: fraude, robos, mentira, falsificación de firmas de los padres. Sin embargo, son más comunes: burlas, bullying, ciberbullying, agresiones, peleas y gestos ofensivos. Todos estos pueden ser tratados desde una fundamentación ética que lleven al estudiante a una reflexión sobre su proyecto personal de vida en su rol como ciudadano.

En respuesta a estos conflictos, la comunidad de indagación como herramienta característica de la pedagogía en $\mathrm{FpN}$, no solo aborda, sino que cuestiona algunos conceptos filosóficos, tanto polémicos como problemáticos,

Lo justo, lo verdadero, lo bueno, la amistad, la belleza, el espacio, el tiempo, personas, reglas, derechos, obligaciones, libertad, identidad, mente, conocimiento etc. Las habilidades de pensamiento son trabajadas en las clases, a partir de los cuentos y novelas, con el aporte de las sugerencias y ejercicios de manuales para el profesor, cuyo autor es Lipman. ${ }^{24}$

Producto del trabajo de FpN con los conceptos éticos anteriormente mencionados, tenemos como rasgos esenciales del pensamiento crítico: lo correcto, lo creativo y lo independiente; al basarse en el buen uso de los criterios y en el perfeccionamiento del razonamiento, es un pensamiento que evalúa y determina todos los elementos implicados en los juicios. Se sustenta en una razón limitada, controlada y contextualizada.

\footnotetext{
${ }^{24}$ ALDANA, Julio: "Comunidad de indagación filosófica. Una alternativa para el fomento de la lectura crítica - reflexiva”, en Ciencias Sociales y Educativas, número 2, Santa Ana de Coro, 2017. Pág. 11.
} 
Finalizando esta última entrevista, el docente afirma que el contenido filosófico se puede tomar de varias maneras para abordar los conflictos que suelen presentarse en la vida escolar. Como preventivos en la medida que se anticipan, que solucionan desde el conocimiento y que podrían convertirse en un análisis de lo hipotético antes de actuar; y como base porque ofrecen un espacio de diálogo para llegar a una conciliación.

El docente es el agente que está directamente relacionado con los niños en el aula de clase, está llamado a ser líder, dinamizador y motivador del conocimiento filosófico; sin él, el programa no podría ser desarrollado. Sus cualidades profesionales y compromiso en la construcción del mismo son determinantes para obtener buenos resultados. Él docente del área es quien lo presenta, lo desarrolla, genera discusión entre sus estudiantes y finalmente trabaja con conceptos, a los que el estudiante acudirá como fuente de solución de muchas problemáticas que se generan en su propio entorno escolar, por eso considero fundamental que los docentes que trabajen con los niños deberán recibir una capacitación previa en FpN.

Lipman afirma que "Los niños empiezan a pensar en el pensamiento, a descubrir en un proceso comunitario de búsqueda los principios del razonamiento y a saber aplicar esos principios que van descubriendo a las situaciones de la vida cotidiana” ${ }^{25}$. Por ende, el maestro debe hacer reconocimiento de esa condición y encargarse de construir casos y anécdotas que puedan aprovechar esa capacidad del niño, orientándolo a tomar decisiones asertivas, en las que el docente consolide habilidades y construya un perfil de estudiante crítico que viva la filosofía.

FpN surgió como respuesta a la carencia conceptual para razonar. El programa, junto a las habilidades de pensamiento crítico que

${ }^{25}$ LIPMAN, Matthew, El descubrimiento..., op. cit., pág. 72. 
Mónica Velasco explicita anteriormente, busca la adquisición del conocimiento filosófico a partir del análisis de casos, desarrollo de manuales y estrategias pedagógicas en las que el maestro mejora la percepción de la realidad circundante y por ende, la madurez en la elaboración de juicios y razonamientos frente a diversidad de situaciones. El estudiante llega a ser parte activa en decisiones o soluciones que se dan luego de acudir a conocimientos previos, que de alguna manera se van actualizando con el desarrollo del programa.

El maestro, en el diario vivir de la institución educativa, está en contacto permanente con los estudiantes. Tiene la oportunidad de conocer muchos de los conflictos que se generan al interior de las aulas, en las horas de descanso e incluso en sus familias. En muchos casos tiene que actuar como juez, en otros tantos como árbitro o mediador. A veces, simplemente de ejecutor de unas directrices trazadas por los Manuales de Convivencia en sus protocolos, que estipulan procedimientos y garantizan el restablecimiento del orden al interior de la institución, dejando inconformidades en algunos de los agentes participantes del conflicto. Esto me motivó a proponer el manejo de las situaciones problemáticas escolares desde un fondo filosófico. Dando al educador un nuevo recurso que, aparte de ser innovador, le permitiría resolver algunas de ellas. Las personas involucradas en una situación conflictiva tomarían una posición activa y responsable en la misma a partir de una reflexión crítica. Esto permitirá llevar un poco de oxígeno al departamento de convivencia escolar que está saturado por los conflictos escolares diarios.

Por lo que se refiere al primer objetivo específico de la investigación, la FpN desarrolla el pensamiento crítico en una ética ciudadana. La norma, como elemento constitutivo de este argumento, debe ser entendida por todos los miembros de la comunidad de indagación, de tal manera que, comprendida con 
cierta base conceptual, ella consiga un abordaje justo de los conflictos, que por un lado, garantice su aplicabilidad universal; y por el otro, tenga en cuenta la contextualización de cada caso en particular. Esta dinámica se debe proyectar siempre hacia una vida cotidiana que fortalezca las relaciones interpersonales de los estudiantes.

En el caso de la institución educativa en la que se aplicó el proyecto, se identifica que han ido trabajando según el modelo pedagógico propuesto del PEI (Proyecto Educativo Institucional). En él se mezclan las siguientes propuestas: el aprendizaje significativo y un enfoque Humanista basado en la axiología del Plantel, lo cual facilita la implementación del programa; "entendiendo los valores como cualidades de las cosas, de las acciones, de las personas, que nos atraen porque nos ayudan a hacer un mundo habitable"26. Estas dos opciones preparan al sujeto para que comprenda ciertas prácticas mediante una reflexión continua de las mismas. Tomadas en conjunto, permiten al niño asociar análisis de casos y contextualizarlos desde el momento de la identificación de sus causas. Esto implica no solo conocimiento, sino influencia ético-ciudadana para garantizar el éxito de FpN. Se puede inferir que los estudiantes son capaces de una ejemplificación de contenidos temáticos y hasta de su conceptualización.

El educador debe, en todo momento, estar atento a las posibles innovaciones curriculares. FpN se convierte en una buena razón para ello. Pues por una parte, debe desarrollar y nutrir sus conocimientos filosóficos, buscar en ellos los argumentos que va a presentar al niño para la solución de conflictos en el interior de la institución educativa; y por otra, desarrolla su creatividad en la medida en que debe empezar a ajustar los mismos con la temática propuesta. Podría tomar del programa de FpN las bases

${ }^{26}$ CORTINA, Adela, Educación en valores..., op. cit., pág. 29. 
orientadoras a la innovación, haciendo que coincidan los contenidos con la realidad que caracteriza el colegio, dándole preponderancia al interés que despiertan los casos que resultan más recurrentes en la cotidianidad.

\section{Afinidad con otros resultados}

El programa de Filosofía para Niños ha sido estudiado como herramienta educativa para favorecer un clima positivo dentro del aula. Destaco el más desarrollado en Chile desde la década de los 90, el de Matthew Lipman, que enfatiza que además del progreso cognitivo, el cultivo de la filosofía por parte de los niños los dispone hacia una valoración de la diversidad y un respeto a todo sujeto. Los niños que hacen filosofía reconocen personas válidas en sus compañeros de aula, aunque piensen distinto, y buscarán acuerdos a partir de los mejores argumentos que surjan en su comunidad de indagación. La filosofía en los niños los orienta hacia la resolución de conflictos mediante acuerdos con fundamentación racional.

Según lo estudiado en Chile, la argumentación es un proceso cognitivo que tiene al menos tres momentos considerados fundamentales.

El primero es el conflicto. La argumentación surge como respuesta a un conflicto, que interpela a los hablantes a resolverlo a través del uso de la argumentación. El segundo es la reconstrucción colaborativa del pensar. La argumentación grupal promueve la exploración de diversos puntos de vistas, la evaluación de la evidencia o de los fundamentos que los sustentan y la deliberación de cuáles son los mejores argumentos y por qué. A través de esta argumentación las personas comparten sus conocimientos, se desafían mutuamente y logran robustecer sus posturas. Por último, la argumentación 
conlleva a examinar las propias teorías y los principios que las sustentan, mejorando la comprensión del proceso de construcción de conocimiento. ${ }^{27}$

En México, Gonzalo Romero y Amparo Caballero proyectan la FpN como un conjunto sinérgico de responsabilidades para desplegar un cambio en el actuar, brindando al estudiante y al docente la práctica de un trabajo transformador. Se trata de un proyecto educativo encaminado a profundizar en los valores de una relación entre personas que se cuestionan por lo que sucede a su alrededor, respetándose, escuchándose y tomando la palabra, para la creación de un clima social de aula positivo. Por tanto, se toma como "una oportunidad para ahondar en el conocimiento y las habilidades sociales que necesitamos para actuar en el conjunto de la sociedad con sentido crítico, bases para una acción transformadora., 28

En su trabajo de campo, ellos observan la presentación de un mundo violento en las aulas como noticia constante de actualidad. El conflicto se aborda de forma individualista, exclusivamente centrado en el estudiante, pasa por alto que el modo en que se configuran las relaciones sociales y cómo se solucionan los conflictos fuera del entorno de los centros escolares, influye poderosamente en la forma en que el alumnado, a su vez, se relaciona con su contexto social.

Al relacionar la aplicación de FpN con la problemática del párrafo anterior, Gonzalo y Amparo se dan cuenta que la racionalidad de la acción comunicativa consiste en la supresión de las relaciones de fuerza, especialmente las preestablecidas en las estructuras de comunicación que impiden la configuración consciente de los

\footnotetext{
${ }^{27}$ EYZAGUIRRE, Sylvia: "El rol de la filosofía en el desarrollo del pensamiento crítico”, en Puntos de Referencia, número 485, Santiago de Chile, 2018. Pág. 4.

${ }^{28}$ ROMERO, Gonzalo \& CABALLERO, Amparo (2008). "Convivencia, clima de aula y filosofía para niños”, en Revista Electrónica Interuniversitaria de Formación del Profesorado, número 27, Guadalajara, 2008. Pág. 6.
} 
conflictos y su regulación consensual por medio de la comunicación interpersonal. "La acción comunicativa ayuda a la renovación de la cultura, así como al logro de la solidaridad y al desarrollo de las identidades personales."29

Referente a los estudios del iraní Mehrnoosh Hedayati, según la tabla ${ }^{30}$ presentada a continuación, la calificación promedio de los estudiantes en habilidades de comunicación interpersonal, tuvo un aumento notable después del tercer grado. La participación de los niños en la indagación filosófica les ayuda a lograr ventajas específicas tales como el incremento de la participación activa en los comportamientos sociales. El cuestionamiento y la participación grupal tienen numerosas ventajas para los niños, porque este método les permite desarrollar sus habilidades sociales, cognitivas, orales, además de habilidades de relación comunicacional, lo que crea condiciones para enfrentar y superar la timidez.

\footnotetext{
${ }^{29}$ Ibíd., pág. 7.

${ }^{30}$ HEDAYATI Mehrnoosh: "Efectos del programa de filosofía para niños a través del método de comunidad de indagación en el mejora de las habilidades de relación interpersonal en estudiantes de primaria” en Childhood \& Philosophy, número 9, Río de Janeiro, 2009. Pág. 211.
} 
Table 2.Follow-up study of persistence effect of treatment after 4 months

\begin{tabular}{|c|c|c|c|c|c|c|c|c|}
\hline & & \multicolumn{7}{|c|}{ Paired Differences } \\
\hline \multirow[t]{5}{*}{ group } & \multirow[t]{5}{*}{ Df } & \multirow[t]{5}{*}{ T } & \multirow{3}{*}{\multicolumn{2}{|c|}{$\begin{array}{l}95 \% \text { Confidence } \\
\text { Interval of the } \\
\text { Difference }\end{array}$}} & \multirow{5}{*}{$\begin{array}{l}\text { Std. Error } \\
\text { Mean }\end{array}$} & \multirow{5}{*}{$\begin{array}{c}\text { Std. } \\
\text { Deviation }\end{array}$} & \multirow[t]{5}{*}{ Mean } & \multirow{5}{*}{$\begin{array}{l}\text { Sig. (2- } \\
\text { tailed) }\end{array}$} \\
\hline & & & & & & & & \\
\hline & & & & & & & & \\
\hline & & & Upper & Lower & & & & \\
\hline & & & Bound & Bound & & & & \\
\hline Third girls & 13 & $-6 / 53$ & $-4 / 06$ & $-8 / 07$ & 192 & $3 / 47$ & $-6 / 07$ & $/ 001$ \\
\hline fourth girls & 11 & $-7 / 50$ & $-5 / 65$ & $-10 / 34$ & $1 / 06$ & $3 / 69$ & -8 & $/ 001$ \\
\hline Fifth girls & 10 & $-4 / 43$ & $-4 / 74$ & $-14 / 08$ & 2/12 & 7.35 & $-9 / 41$ & 1001 \\
\hline Third boy & 11 & $-4 / 62$ & $-2 / 62$ & $-7 / 37$ & $1 / 08$ & 3/74 & -5 & $/ 001$ \\
\hline fourth boy & 11 & $-4 / 77$ & $-3 / 50$ & $-9 / 49$ & $1 / 36$ & $4 / 71$ & $-6 / 5$ & 1001 \\
\hline Fifth boy & 10 & $-9 / 39$ & 187 & $-2 / 14$ & 167 & $2 / 24$ & $-6 / 63$ & $/ 001$ \\
\hline
\end{tabular}

“La filosofía para niños está diseñada para adaptarse a las necesidades de los niños, está presentada en historias que conectan los significados de la vida lógica, estética y moral”31. Mehrnoosh Hedayati tiene como objetivo estudiar a los niños y sus reacciones hacia ideas relacionadas con la ética y la política como el respeto a los padres, la libertad, negociación, juicio, igualdad y justicia. Él encuentra que este estudio le da a los estudiantes una oportunidad amplia de acoplar sus propias acciones con sus ideas y de aprender hábitos de pensamiento reflexivo, respetando las ideas de otras personas y colaborando en su evaluación, para perseguir un compromiso saludable, ejercitar el autocontrol y cultivar el buen juicio.

La investigación de Hedayati concluyó que la participación en Filosofía para Niños podría continuar su efecto positivo en la comunicación interpersonal de los estudiantes. Basado en la tabla, las habilidades comunicativas son mucho más palpables al menos

${ }^{31}$ Ibíd., pág. 203. 
después de 4 meses de su aplicación, tanto en mujeres como en hombres, en cada uno de los últimos 3 grados de básica primaria. El estudio para la evaluación del pensamiento filosófico, celebró una hora de FpN durante 16 semanas en el primer año, dejando abierta la posibilidad de hallar otros resultados positivos a mediano o a largo plazo. Como conclusión de estas sesiones se encontró un aumento en la capacidad del estudiante en conductas verbales y no verbales, y una mejora en el razonamiento lógico.

\section{Conclusiones}

Filosofía para Niños $(\mathrm{FpN})$, al implementarse dentro de una institución educativa que fundamenta su acción pedagógica en valores, y desde el PEI ofrece un modelo pedagógico humanista. Es una oportunidad, no solo para iniciar al estudiante en habilidades de pensamiento crítico, sino también para la aplicación de las mismas en la búsqueda de soluciones a conflictos escolares que hacen parte de la cotidianidad. Esto, teniendo en cuenta que el programa no deja de ser un elemento significativo de retroalimentación y renovación permanente en el ejercicio de una ética-ciudadana.

La aplicación de las estrategias metodológicas que integran el programa FpN debe ser alcanzable por el docente a cargo, debido a que verificar el cumplimiento del proyecto pasa por su responsabilidad y compromiso ante lo que previamente se ha hecho comprensible para él. Además es él quien le hace seguimiento para identificar los resultados, retroalimentarlos y fortalecerlos, orientando lo que el estudiante demuestra en su cotidianidad escolar frente a sus relaciones interpersonales.

Es pertinente la propuesta de FpN incorporada al Área de Ética y Valores. Ésta introduce diversas estrategias que le permiten al niño descubrir, desde el mismo momento en que se conforma una 
comunidad de búsqueda, la importancia del análisis de situaciones problémicas. La afectación en el conflicto, sea familiar o escolar, puede abordarse inicialmente con la reconstrucción del hecho en forma anecdótica, analizando los casos con la metodología que presentan los textos escritos por Lipman, incluyendo los Manuales del Docente. Habitualmente el niño se identifica con las narraciones o con una lectura orientada por el maestro, lo cual especifica cada situación, motivándolo a cuestionar y cuestionarse, enseñándole a categorizar y a construir un diálogo del que se generan unas conclusiones. En este proceso se gestan habilidades de pensamiento que contribuyen a formar en él un carácter crítico. Tener la oportunidad de atestiguar los primeros frutos en niños y niñas que poco a poco se van haciendo más críticos y objetivos, reafirma la necesidad de conocimientos filosóficos en el aula, pues ellos van dando al niño herramientas cognitivas para argumentar y construir soluciones frente a conflictos escolares. A partir de allí, se construye con ellos una verdadera comunidad de diálogo, donde la intervención de todos los miembros es respetada, es decir, hay un desarrollo ciudadano fundamental para encarar problemas cotidianos, permitiendo una atención oportuna y eficaz por parte de lo que ellos mismos han aprendido con FpN.

Filosofía para Niños (FpN), en el área de Ética y Valores, permite incorporar experiencias significativas que acercan a los estudiantes al análisis de situaciones conflictivas que surgen en la cotidianidad escolar. Los ayudan a la búsqueda de alternativas de solución que satisfagan cada una de las partes implicadas en el conflicto, propiciando hechos donde impere el sentido ético; es decir, el cumplimiento libre y consciente de la norma ciudadana, así como la apropiación de la misma, garantizando la formación de mejores ciudadanos.

Para los docentes del área de Educación Ética y en Valores, este proyecto se convirtió en un reto, puesto que, dentro del contexto filosófico, requiere del ajuste necesario para que se constituya en 
un verdadero aprendizaje significativo, al intentar asociar lo que el estudiante ya poseía, con lo que se pretende adquirir. Aterrizado en la ética ciudadana, contribuye a la moralización del individuo, es decir, le permite remitirse al conocimiento filosófico para asumir las diversas problemáticas, pues en principio, corresponden al ambiente escolar, pero estas estarán presentes en su futuro desempeño laboral, familiar y profesional. Debido a esto, se obtiene como resultado un individuo más justo y proactivo en la solución de sus propios conflictos sociales. Todo esto colabora asertivamente en la formación de la conciencia moral.

El conflicto escolar estará vigente en una institución educativa, siempre atrae la atención de quienes integran la comunidad educativa. Ello permite al docente categorizarlos, convertirlos en objeto de estudio de su clase, para desde estos, acudir al conocimiento filosófico con el fin de buscar causas, consecuencias, soluciones, involucrados, alcances, ofreciendo la oportunidad de adquirir madurez del pensamiento en el abordaje de situaciones problema al interior del aula y de la institución, lo que genera estabilidad y mejor clima de trabajo. Esto se ve reflejado en el componente de ambiente escolar, que es un factor tenido en cuenta para la evaluación gubernamental del establecimiento. Filosofía para Niños $(\mathrm{FpN})$, garantiza de esta manera, la participación activa del estudiante en la vida institucional, como ideal que se propone desde la propia legislación colombiana.

En todos los momentos de la vida escolar y en el aula de clases, el trabajo ininterrumpido de $\mathrm{FpN}$ se convierte, para el maestro y para la institución, en una buena práctica pedagógica; les permite evaluar frecuentemente el impacto de su implementación. Así mismo, introduce a una reflexión permanente sobre el quehacer del educador y la formulación, o reformulación de estrategias que contribuyan a generar en el estudiante un compromiso ético con sus conocimientos y con el entorno de sus compañeros. Favorece la 
solución de conflictos y la formación en valores ciudadanos en la institución donde se aplique el proyecto.

\section{Bibliografía}

ALDANA, Julio: "Comunidad de indagación filosófica. Una alternativa para el fomento de la lectura crítica - reflexiva”, en Ciencias Sociales y Educativas, número 2, Santa Ana de Coro, 2017. Págs. 8-15.

CORTINA, Adela: Educación en valores y Responsabilidad cívica, Editorial el Búho Ltda, Bogotá, 2005.

CORTINA, Adela: "Resolver los conflictos, hacer la justicia”, en Cuadernos de pedagogía, número 257, Madrid, 1999. Págs. 1-4. EYZAGUIRRE, Sylvia: "El rol de la filosofía en el desarrollo del pensamiento crítico", en Puntos de Referencia, número 485, Santiago de Chile, 2018. Págs. 2-7.

HEDAYATI Mehrnoosh: "Efectos del programa de filosofía para niños a través del método de comunidad de indagación en el mejora de las habilidades de relación interpersonal en estudiantes de primaria” en Childhood \& Philosophy, número 9, Río de Janeiro, 2009. Págs. 199-215.

HOYOS VALDÉS, Diana: "Filosofía para niños y lo que significa una educación filosófica”, en Discusiones Filosóficas, número 16, Manizales, 2011. Págs. 149-167.

LIPMAN, Matthew: El descubrimiento de Harry, Ediciones de la Torre, Madrid, 1988.

LIPMAN, Matthew: Investigación ética - Manual del profesor para acompañar a Lisa, Ediciones de la Torre, Madrid, 1988.

MORENO ARTIDIELLO, Mabel Marta: "Filosofía para Niños y Niñas (FPNN): una oportunidad diferente para pensar en la escuela”, en Ciencia y Sociedad, número 43, Santo Domingo, 2018. Págs. 25-38. 
NAJÍ, Saeed: "Filosofía para niños entrevista a Matthew Lipman”, en Tedium Vitae, número 2, Guadalajara, 2012, págs. 2-12. ROMERO, Gonzalo \& CABALLERO, Amparo (2008). "Convivencia, clima de aula y filosofía para niños", en Revista Electrónica Interuniversitaria de Formación del Profesorado, número 27, Guadalajara, 2008. Págs. 2-7.

VELASCO, Mónica: "Filosofía para niños: una alternativa para resignificar la práctica”, en Sinéctica, número 8, Guadalajara, 1996. Págs. 1-12.

ZULETA, Estanislao: Educación y democracia, un campo de 